\title{
Chronic Disease Self Management Using a Social Networking PHR/UHR
}

\author{
Jeremy S. KAGAN ${ }^{1 a}$ \\ ${ }^{a}$ Corporate, CardioLync, Israel and USA
}

\begin{abstract}
This viewpoint paper presents a potential solution to the "information islands" that are holding back PHR/UHR from becoming truly effective diagnostic information care management tools for patients especially those who suffer from chronic diseases. The solution involves integrating patient portal with a diagnostic data interface layer to create a single access point for caregivers and patients.
\end{abstract}

Keywords. Digital Health, PHR, UHR, PACS, EHR, Diagnostic Images, Diagnostic Data, Interpretive Reports, Patient Portal

\section{Introduction}

Aging populations, increased prevalence of chronic conditions, and commensurate rising costs significantly challenge health care systems worldwide. One proposed solution to these challenges has been health information technologies (ITs) that empower patients to be partners in their care, and that support evidence-based individualized care. But there is a tendency to downplay the complexity of implementation and user adoption [1]. Patients who suffer from chronic disease often have multiple concurrent chronic conditions and complications that require regular visits with a number of different specialists in addition to their primary care physician (PCP). They also may have intermittent interactions with emergency rooms and other care settings. This puts them at increased risk for severe adverse events if information does not flow between care settings timely and accurately.

Personal Health Records (PHR) together with Universal Health Records (UHR) can mitigate this problem. PHR Allows patient to maintain all their care information as well as uploaded data from personal monitoring devices, health smartphone apps, and smart home devices like medical toilets, and daily diaries of their wellbeing. UHR is Patient-centered information available to and often controlled by patients, that contains all health care information and history. A PHR combined with a UHR would drastically reduce the risk that care-providers may overlook pertinent information.

Despite massive effort and investment in health information systems and technology, and many years of widespread availability, the reality is that most physicians still have to fax and mail patient records the way they did a decade ago [2]. A fragmented system of storing and retrieving essential patient data impedes optimal care. If they cannot exchange data with other health care systems, PHRs will become "information islands" that contain subsets of patients' data, isolated from other information about patients, with limited access and transient value [3]. When PHRs are

\footnotetext{
${ }^{1}$ Corresponding author, Jeremy S. Kagan, email: jeremy.kagan@cardiolync.com
} 
integrated with EHR systems, they provide greater benefits than would stand-alone systems for consumers.

This viewpoint paper presents an innovative solution to the "information islands" for combined PHR/UHR to become a truly effective care management tool for patients. The solution involves integrating patient portal with a cross-modality diagnostic data interface layer to create a single integrated access point for caregivers and patients.

\section{Methods - Integrated Access Point for Care-Givers and Patients}

Effective communication and coordination among doctors, specialists and other caregivers could mean the difference between life and death for patients. A crosssystem data interface layer can integrate images and data across diagnostic modalities, and thus simplify the process of accessing information by doctors for medical interpretations, reporting and treatment recommendations, and aggregate this information for patients. Diagnostic interface layer technology can help care providers improve patient outcomes by facilitating initial risk stratification and remote consults with experts, thereby reducing admissions and readmissions [4].

Since the adoption of PACS in the late nineties and early 2000s, imaging exams have been stored digitally. There is a misconception that digital imaging helped medical image exchange eliminate any potential image loss. Unfortunately, this is not the case: despite the most recent advances in digital imaging, most hospitals still often lose their imaging data, with these losses going completely unnoticed. As a result, not only does image loss affect the faith in digital imaging but it also affects patient diagnosis and daily quality of clinical work [5].

Furthermore, when patients travel from provider to provider, image availability at the point of care can be a problem. Patients may receive CDs with copies of their imaging studies, but there is risk that this fragile media could be damaged and become unreadable. CDs also can be easily misplaced or lost by the patient, and some patients simply forget to bring their CDs to the appointment. At the receiving end, images are sometimes viewed using the viewer on the $\mathrm{CD}$, but more commonly copied from the $\mathrm{CD}$, updated with local patient and order information, and loaded into the local PACS. This requires significant manual local effort.

Electronic exchange provides opportunities for improved operational workflows that can positively impact patient care, reduce cost, improve patient and clinician satisfaction, and can even increase revenue opportunities in key service lines. CDbased image exchange has laid an important foundation to support the emerging next generation of interoperable, standards-based image exchange [6].

A data interface layer can support standardized integration of clinical technologies using existing industry standards to facilitate automatic upload of images and data. Using DICOM file exports from all modalities such as ECG/EKG, ECHO, ultrasound, CT, PET, MRI, nuclear imaging, it is possible to interface directly to the diagnostic machine or to the PACS for the diagnostic data, and to the EHR system with interpretation results in HL7. A data interface layer can also accept data from Holter monitors, mobile telemetry apps, home patient follow-up visit reports for monitoring and evaluation. It allows for data from patient monitoring devices, smartphone apps, and future tools such as Artificial Intelligence algorithms. By converting files of images, clips, and data from DICOM or other modality specific formats into web friendly jpeg, mp4, and PDF formats, a data interface layer can provide single point 
access from any computer without installation of special client software like a DICOM reader or modality specific application, and can sit atop PACS systems for messaging and reporting via the web.

\section{Results - Social Networking Paradigm}

Social networking may be the answer. It is a proven communication and coordination model that can be applied to facilitate interdisciplinary and cross-institution imaging interpretation and expert consults on a UHR. Social networking can be implemented via a web-based interface portal to link doctors, diagnostic imaging techs, experts and patients for remote diagnosis; and to speed access to images, data, and history; which ultimately cuts the time to the correct treatment plan.

The social networking communication and information sharing medium has the power to revolutionize the way physicians interact with their patients and fellow health care workers. When managed correctly, it can provide a great way for doctors to communicate and educate others. For example, a surgeon used Twitter during a robotically assisted partial nephrectomy to let other surgeons know that a total nephrectomy was not necessary, despite the large tumor size [7].

A patient portal that creates a secure social network of physicians with patients is a clinical tool that allows a primary care physician to retain interaction with the patient and seek specialty consult when they jointly determine the need together. This empowers patients to take a more active role in their treatment and saves money.

Online shared access to critical data is secured by pre-authorized user restrictions and permissions. Single access to all diagnostic and history event data facilitates remote interpretation and reporting by specialists. Using an interface layer, diagnostic tests can be administered locally by a tech, and then be interpreted by a specialist who may be sitting remotely. Pre-screening evaluation can determine if the patient needs to travel for a physical exam or procedure, thus reducing referrals and hospital admissions.

\section{Discussion}

In addition to access, the integrated patient portal and data interface layer can provide a private messaging platform for secure communication among doctors, experts, technicians and patients. Table 1 shows the main advantages of the integrated interface portal compared to the current state of the art, as observed by the author in clinical use of an implementation of the integrated interface portal.

Table 1. Observed Advantages of the Integrated Interface Portal model

\begin{tabular}{lll}
\hline $\begin{array}{l}\text { Integrated } \\
\text { Interface Portal }\end{array}$ & $\begin{array}{l}\text { Pitfall that it } \\
\text { Addresses }\end{array}$ & What it Provides \\
\hline $\begin{array}{l}\text { Social Networking } \\
\text { Model with History }\end{array}$ & $\begin{array}{l}\text { Communication } \\
\text { Access } \\
\text { Population Coverage }\end{array}$ & $\begin{array}{l}\text { Improves care coordination among doctors and other experts } \\
\text { to cut decision time and drives engagement with patients via } \\
\text { access to history and recommendations at point of care }\end{array}$ \\
\hline $\begin{array}{l}\text { Structured } \\
\text { Reporting }\end{array}$ & Workflow & Speeds the workflow for the Interpreting Physician \\
\hline
\end{tabular}




\begin{tabular}{lll}
\hline $\begin{array}{l}\text { Single Access for } \\
\text { data and images }\end{array}$ & Interoperability & Physicians have access to review studies \\
\hline Patient Portal & Patient Engagement & Encourage consults and access in emergencies \\
\hline $\begin{array}{l}\text { Private caregiver } \\
\text { Messaging }\end{array}$ & Coordination & $\begin{array}{l}\text { Secure communication by and between doctors, specialists, } \\
\text { and technicians for clarifications }\end{array}$ \\
\hline
\end{tabular}

\subsection{A Model that Keeps the Patient in Center Focus}

The integrated interface portal consolidates information- data, measurement, history, and images; and facilitates sharing of that information among caregivers and patients at the point of care and remotely. The social networking model places the patient squarely in control of their condition with their individual timeline of medical events. The patient portal functionality gives patients access to their history and reports so that they can seek other expert advice as well as never be caught short in an emergency.

The level of information that patients can upload themselves or access can be limited by their primary care provider or other entity that manages their diagnostic data. As far as the patient's caregivers are concerned, questions and answers between the doctors and specialists can remain private and not available to the patient. In cases where a primary care physician is advised to have a psychologist present when presenting an expert's findings and opinions, they can limit patient access to these reports until after the primary care physician discusses them directly with the patient.

In addition, with the right permission settings, an integrated interface portal can best fulfill the role of a UHR, as it is patient-centered, and the information available can be controlled. Similarly, as the portal interfaces to diagnostic modalities like ultrasound, nuclear imaging, and PACS systems; technically the portal can just as effectively interface with other portals of the patient's various health care providers. If there is an operational or regulatory mandate available that allows the sharing of PHI (private health information) by and between healthcare organizations, as per a patient's permission, then all of the patient's data and history can be accessed via the portal.

This would eliminate the major shortcoming of most patient portals today, namely, data access across multiple organizations and institutions. With an integrated interface portal, patients and caregivers will have a non-fragmented view of patient data.

Similarly, the interface portal model can facilitate a patient's PHR, by allowing patients to maintain all of their care information as well as upload data from personal monitoring devices, smartphone apps, smart home devices, and daily wellness diaries.

PHRs can be set so that if any of the patient/home device generated data exceeds a certain pre-defined threshold, the patient's primary care physicians can be automatically notified. This would make it easier for caregivers to maintain continuous communication with patients, not just episodic, and to track chronic conditions and illnesses and post-discharge follow-up so that they could enact early interventions.

Technology takes time to be deployed in hospitals and healthcare networks, and introducing a new technology will require clearly defined and demonstrable added value. An interface portal offers such added value. PACS system vendors continually expand the features and use cases of their systems, but the applications were built to meet the needs of specific specialties and disciplines which restrict them. An integrated interface portal on the other hand introduces a layer that sits atop of and connects all PACS, EMR, and native diagnostic modalities for requisite access and/or sharing. 
Organizing patient information around a single access point enables specialists to remain focused on analyzing the data and translating it into an appropriate plan of care. This is a vast improvement over current inefficiencies whereby doctors log in to multiple systems to interpret various test results, which could lead to frustration and possible distraction from their primary focus on the patient's plan of care.

The social networking aspect improves patient care by connecting the principal people involved with the necessary and relevant information. It consolidates patient medical diagnostic tests, ongoing monitoring, and event data from multiple sources and enables a consolidated visualization in a timeline shared with patient and caregivers. Doctors can interpret native diagnostic images, and encourage patients to securely access and update their medical histories when they experience health related events.

The author has led a team that implemented this model in a technology which is in clinical use. Each type of user: doctor, technician, patient has specific permissions for what they are able to access, view, upload and download.

\section{Conclusions}

An integrated interface portal presents patient health events, diagnostic data and images; and creates social networking interaction between patients and physicians for communication, questions and answers about a patient's specific chronic condition and history. Primary care physicians and patients alike can use the interface portal alike to trigger and facilitate teleconsults with specialists and subspecialists.

The integrated interface portal allows specialists who may be located remotely to access all relevant medical material including patient history, data and images and have these data before their eyes in an integrative and systemic manner; while engaging patients with controlled but direct access. This improves joint decision-making and encourages patients to more effectively self manage their chronic conditions.

\section{References}

[1] Avdagovska M, Stafinski T, Ballermann M, Menon D, Olson K, Paul P. Tracing the Decisions That Shaped the Development of MyChart, an Electronic Patient Portal in Alberta, Canada: Historical Research Study. Journal of medical Internet research. 2020; 22(5): e17505.

[2] Reisman M. EHRs: The Challenge of Making Electronic Data Usable and Interoperable. P \& T : a peerreviewed journal for formulary management. 2017; 42(9): 572-575.

[3] Tang PC, Ash JS, Bates DW, Overhage JM, Sands DZ. Personal health records: definitions, benefits, and strategies for overcoming barriers to adoption. Journal of the American Medical Informatics Association JAMIA. 2006; 13(2): 121-126. https://doi.org/10.1197/jamia.M2025

[4] Kagan JS. Doctor-Patient Social Networking to Improve Specialist Care Coordination. In McHaney R W, Reychev I, Azuri J, McHaney ME, \& Moshonov R. (Eds.) Impacts of Information Technology on Patient Care and Empowerment (pp. 267-289). 2020 IGI Global. http://doi:10.4018/978-1-7998-00477.ch014

[5] Oglevee C, Pianykh O. Losing Images in Digital Radiology: More than You Think. J Digit Imaging 2015; 28: 264-271. https://doi.org/10.1007/s10278-014-9748-2

[6] Vreeland A, Persons KR, Primo HR, Bishop M, Garriott KM, Doyle MK, et al. Considerations for exchanging and sharing medical images for improved collaboration and patient care: HIMSS-SIIM collaborative white paper. Journal of Digital Imaging. 2016; 29(5): 547-558.

[7] Prasanna PM, Seagull FJ, Nagy P. Online social networking: a primer for radiology. Journal of Digital Imaging. 2011; 24(5): 908-912. 